\title{
Modeling the Role of Arterial Windkessel in the Enhancement and Synchronization of Low Frequency Vasomotor Activity
}

\author{
G Baselli ${ }^{1}$, A Porta $^{2}$, M Pagani $^{3}$ \\ ${ }^{1}$ Bioengineering Department, Polytechnical University of Milan, Italy \\ ${ }^{2}$ Department of Preclinical Sciences, LITA Vialba, University of Milan, Italy \\ ${ }^{3}$ Internal Medicine I, "L.Sacco” Hospital, University of Milan, Italy
}

\begin{abstract}
Arterial windkessel mechanisms and arterial pressure (AP) low frequency (LF) waves were investigated by means of simple lumped models of a compliant resistant/arterial tree and of flow regulation in peripheral vascular districts (PVDs) with three types of feedback: 1) delay, 2) Van der Pol oscillator, 3) relay; all were able to actively compensate flow changes and to simulate peripheral LF vasomotion. Each PVD connected to a windkessel compartment displayed a reduction and a disappearance of oscillations with low compliance, when the windkessel equivalent time constant $T_{\text {eq }}$ fall below $2 s$. Two PVDs connected to the same windkessel tended to phase opposition with a negative interference canceling their LF oscillations from AP. With a modest neural drive, cancellation was imperfect and AP waves appeared. Vasomotion, arterial compliances and neural triggers are all essential in forming LF AP variability
\end{abstract}

\section{Introduction}

Cardiovascular variability is the result of complex interactions between central circuits, pressure regulation mechanisms and vascular activity controlling peripheral flow $[1,2]$. However, the role played by basic features of the arterial tree distribution system in enhancing or damping the effects of vasomotion on systemic arterial pressure (AP) and in promoting either positive or negative interferences has not been investigated yet.

The present work addresses the coupling of arterial compliances and windkessel mechanisms with low frequency (LF, $0.1 \mathrm{~Hz}$ ) vasomotion by means of simple models of peripheral blood flow control in peripheral districts connected to a resistant/compliant arterial tree, which is sketched in Fig.1a.

The windkessel effect [3] is a core concept in studying arterial circulation as it explains the buffering action of arterial compliances, which transform the pulsatile aortic flow into a smooth peripheral flow and pressure. This mechanism in its most simple version (disregarding inertances, pressure wave transmission, reflections, etc.) is described by the two element lumped model of Fig. 1b: the peripheral vascular bed is lumped into the total peripheral resistance (TPR) and arterial compliances are represented by $C$. At a given heart rate (HR), the windkessel time constant, $\mathrm{T}_{\mathrm{wk}}=\mathrm{C} \cdot \mathrm{TPR}$, controls the ratio between systolic and diastolic AP, SAP/DAP, and limits pulse pressure.

The concept of a lumped TPR was fruitfully and widely applied not only to the analysis of AP wave but also to the modeling of the baroreflex AP regulation and following this perspective, consequences were drawn relevant to the formation of LF AP waves [4, 5]. However, in this way, important aspects of the arterial

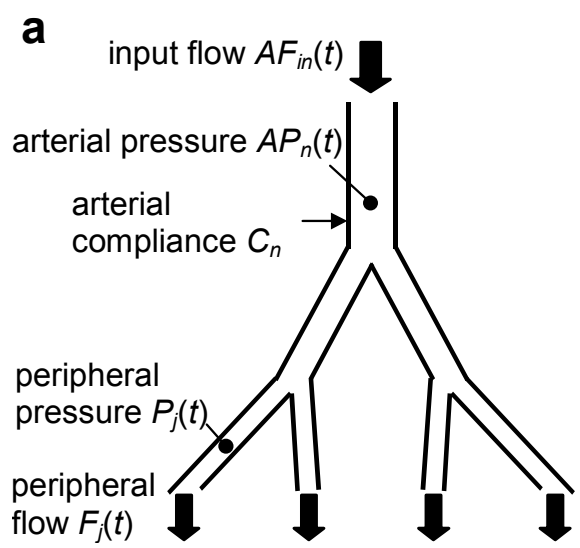

b

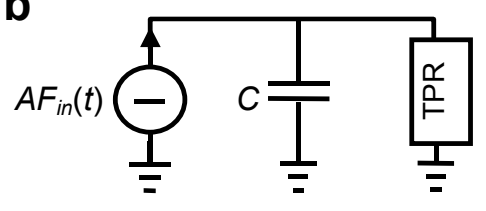

C

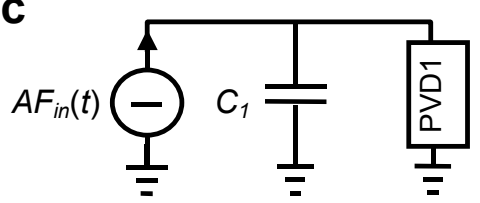

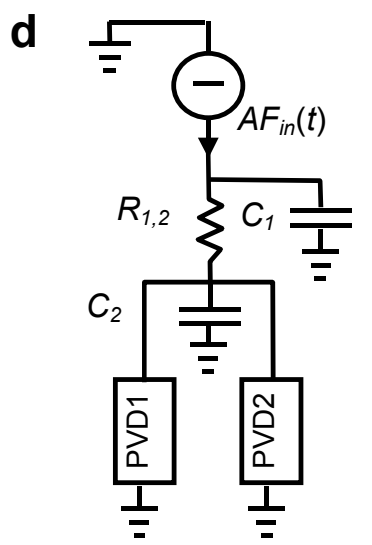

Figure 1. a) Sketch of arterial tree. b) Classical windkessel. c) PVD fed by a windkessel compartment. d) Two PVDs. 
tree conceived as a transport system may have been overlooked. Namely, AP control is only a component of distributed and hierarchically organized vascular regulation mechanisms, whose principal targets should include the maintenance of the optimal flows in the single limbs and organs as well.

The competition of flow control in the many peripheral districts can be assumed to create interferences with and throughout the windkessel compartments of the arterial tree. The aim of the present modeling work is to explore the bases of these interactions analyzing whether they contribute in transferring the well known LF vasomotor activity to the formation of LF AP waves at systemic level, without and with a triggering activity of neural control mechanisms conveying LF waves to vessels through the sympathetic outflow.

\section{Modeling methods}

Local regulation of flow in a peripheral vascular district (PVD) was modeled by a non linear feedback modulating local conductance. The feedback input is based on the cube of a flow deficit computed as the ratio
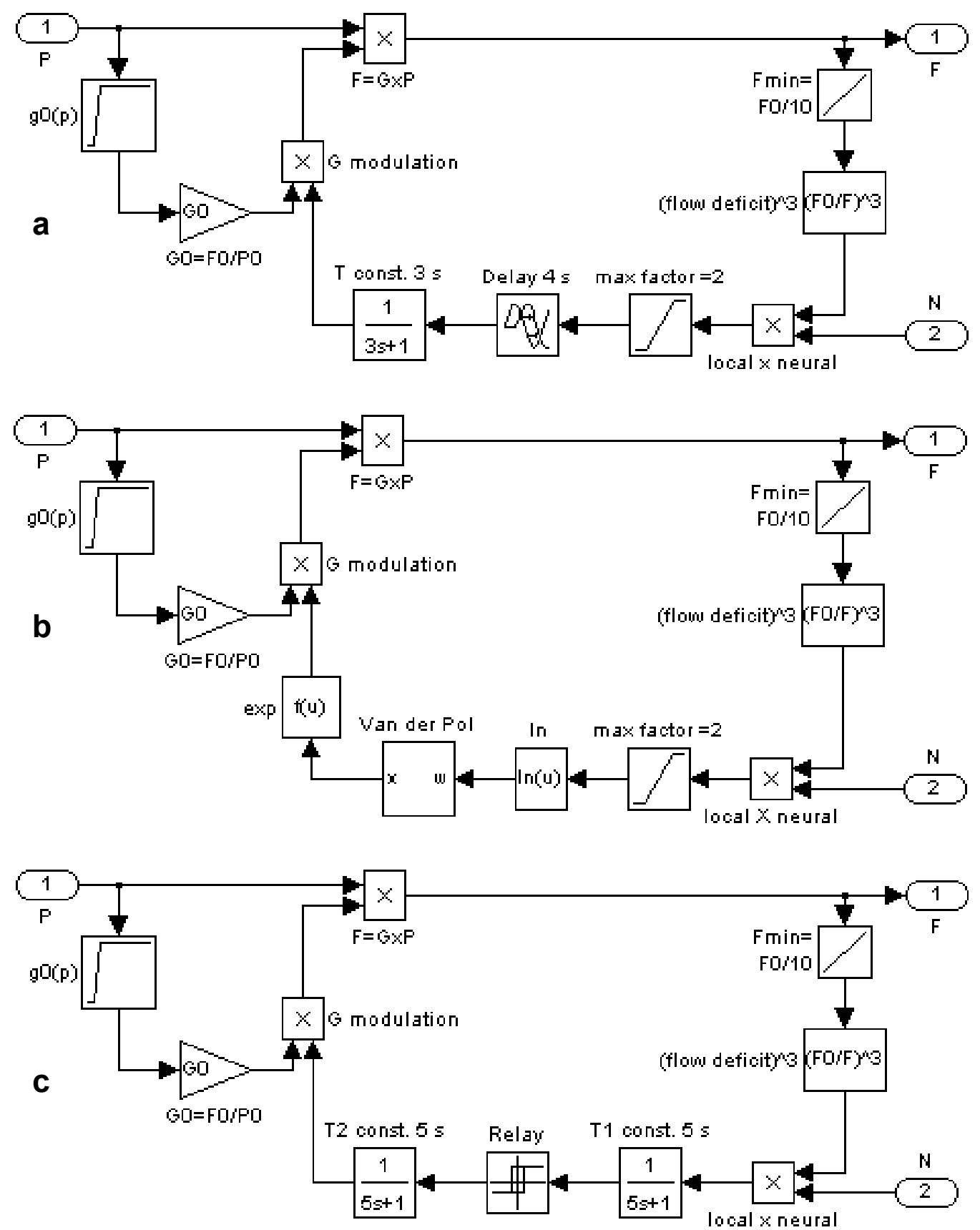

Figure 2. PVDs with different feedback types: a) delay; b) Van der Pol oscillator (see detail in Fig.3): c) relay. 
between the required flow, $F_{0}$, and the actual one $F_{j}(t)$. For generality, various mechanisms were included in the feedback, obtaining different PVD models, displayed in Fig.2: a) a delay of the smooth muscle (pure delay and time constant); b) intrinsic vascular oscillations (van der Pol oscillator, see the detail in Fig.3); c) hysteresis of myogenic responses (relay and time constant). The PVD conductance $G_{j}(t)$ is modulated around the nominal value $\mathrm{G}_{0}$ by the feedback, so that a flow deficit causes vasodilation. Finally, $\mathrm{F}_{\mathrm{j}}(\mathrm{t})$ is the product of the driving pressure and conductance.

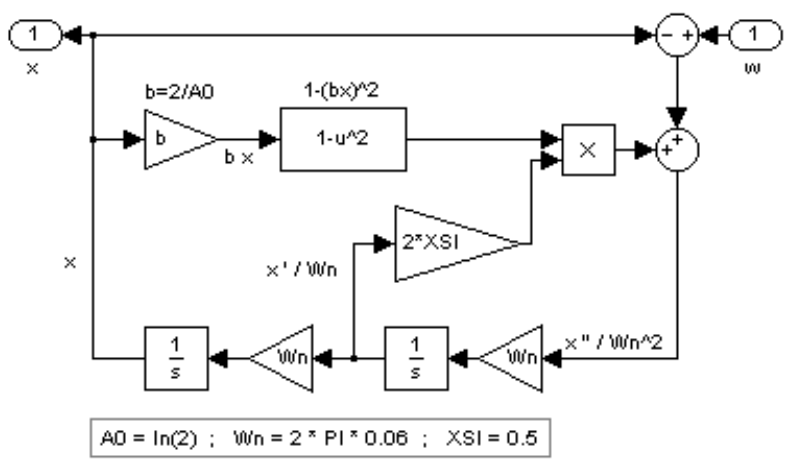

Figure 3. Detail of the Van der Pol oscillator of Fig.2b.

Models were built with the Matlab graphic interface Simulink and simulations carried out with the standard ode45 integration algorithm. The arterial tree was simulated assigning a compliance $C_{n}$ to each node $n$ (node pressure derivative, $\mathrm{P}^{\prime}{ }_{n}(\mathrm{t})=1 / \mathrm{C}_{\mathrm{n}}$ (flow balance)) and a resistance $R_{n m}$ to each branch $n-m$ (branch flow, $F_{n m}=1 / R_{n m}\left(P_{n}-P_{m}\right)$. The input flow, $F_{\text {in }}(t)$, was either constant or a square wave with a $20 \%$ duty cycle $\left(200^{\circ} \mathrm{ms}\right.$ systole, $800 \mathrm{msec}$ diastole); its average was generally balanced to $\mathrm{JF}_{0}$ ( $\mathrm{J}$ number of districts); only with the relay PVD this value had to be augmented by a $6.7 \%$. All extensive quantities are normalized by the arbitrary PVD size given by $\mathrm{F}_{0}$.

Branch resistances and the PVD nominal conductances were dimensioned in order to impose a $100 \mathrm{mmHg}$ mean AP, subdivided into a $60 \mathrm{mmHg}\left(\mathrm{P}_{0}\right)$ at the PVD and equal pressure drops on each branch. Compliances were dimensioned keeping the same total compliance at each branching level (subdivided into many small compliances as the branching order increases) and adapting the compliance size in order to obtain a pulse of $120 / 80$ $\mathrm{mmHg}$, that corresponds to that of a windkessel with $\mathrm{T}_{\mathrm{wk}}=2 \mathrm{~s}$. In this condition an equivalent time constant of $\mathrm{T}_{\mathrm{eq}}=2 \mathrm{~s}$ is assumed: the arterial tree thus adapted to the main windkessel function of pulse buffer was tested as to its interference features with the LF oscillations of the PVDs. In the simple case of Fig.1c (a single windkessel compartment with the passive resistance substituted by an active PVD) the time constant can be readily approximated by $\mathrm{T}_{\mathrm{eq}}=\mathrm{C}_{1} \cdot \mathrm{G}_{0}$; in this way it was possible to explore a wide range of $\mathrm{T}_{\mathrm{eq}}$ values simply by varying
$\mathrm{C}_{1}$. The two PVD models of Fig.2d were dimensioned with $\mathrm{P}_{0}=60 \mathrm{mmHg}$, mean $\mathrm{AP}=100 \mathrm{mmHg}, \mathrm{C}_{1}=\mathrm{C}_{2}$, $\mathrm{T}_{\mathrm{eq}}=2 \mathrm{~s}$.

\section{Simulation results}

All models displayed a range with active flow compensation against AP changes in which self-sustained oscillations at LF appeared. In all the three PVD types this range was quite extended around the nominal $\mathrm{P}_{0}$ and showed oscillations with a $10 \mathrm{~s}$ period between approximately $2 \mathrm{~F}_{0}$ and $0.5 \mathrm{~F}_{0}$.
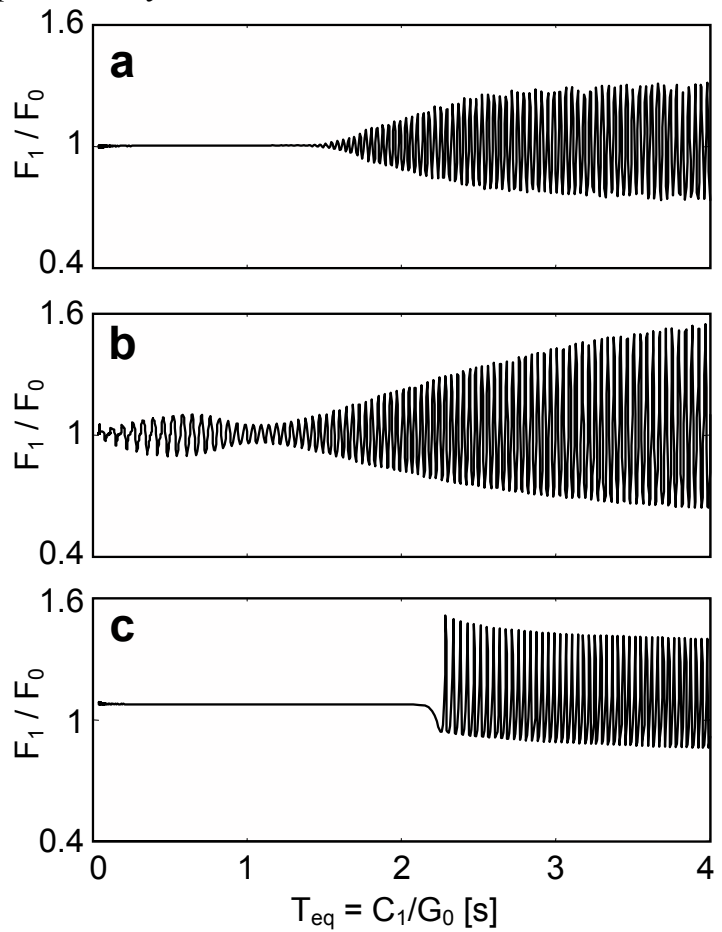

Figure 4. Single PVD models of Fig.2c: a) delay PVD, b) Van der Pol PVD, c) relay PVD. LF oscillations in the PVD flow at varying $\mathrm{T}_{\mathrm{eq}}$ values.

Sufficient arterial compliances were shown to be necessary to allow rhythmic activity. As shown in Fig.4, in all the 3 models LF oscillations decreased to complete extinction for $T e q<2 \mathrm{~s}$. This indicates that a windkessel stiffer than required for the primary function of conditioning the pulse pressure has also a secondary effect in blunting vasomotion.

Further simulations demonstrated a general tendency to phase opposition with 2 PVDs coupled to a common feeding compliance and to maximum phase dispersion with more. The case of 2 PVDs (model of Fig.2d) is displayed in Fig.5. This simulation was carried out by initializing the two identical PVDs to almost equal values with a small difference of 1/1000. Accordingly the oscillations in the PVDs are started in phase; however, this is proven to be an unstable condition which is rapidly lost in favor to phase opposition which is reached within the first $50 \div 80 \mathrm{~s}$ of the simulation. In all the three PVD 
types, phase opposition permits larger peripheral flow oscillations; however, they are hidden by their negative interference both at central $\left(\mathrm{P}_{1}\right)$ and at peripheral $\left(\mathrm{P}_{2}\right)$ AP level (the residual ripple at double frequency is an artifact of the model symmetry).

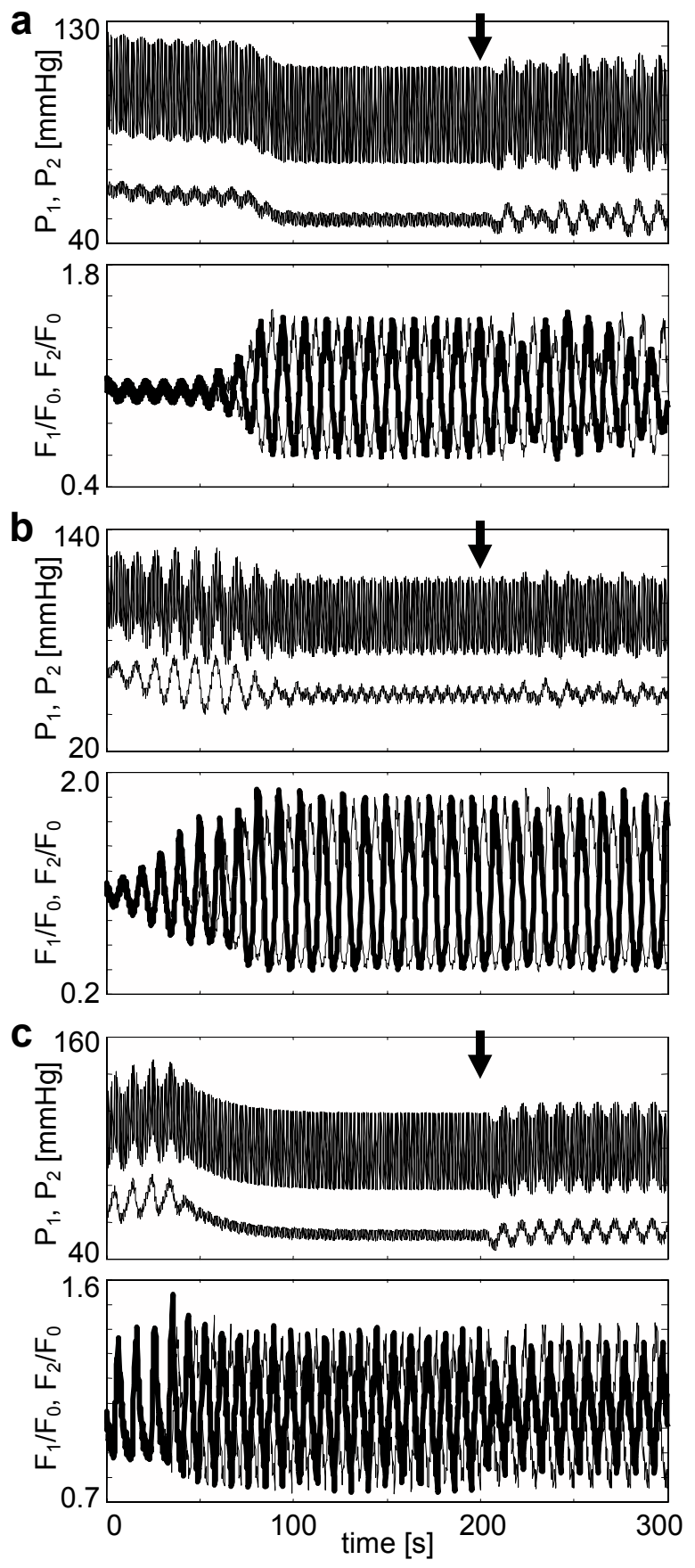

Figure 5. Two PVD models of Fig.2d: a) delay PVD, b) Van der Pol PVD, c) relay PVD. Spontaneous transition to phase opposition of flows (lower panel, heavy and light lines) and cancellation of LF AP waves (upper panels). At $\mathrm{t}=200 \mathrm{~s}$ (arrows) a LF neural drive disturbs phase opposition and unmasks LF AP waves.
This behavior can be demonstrated to rely on basic properties of the interactions with the feeding windkessel compartments, in fact any phase delay is amplified by the load of the preceding PVD (or PVDs) and the maximum distribution efficiency is obtained with sparse phases. Also the large peripheral oscillations permit a reduction of the power needed to maintain the mean flow [6].

Up to now no neural drive was present, $\mathrm{N}(\mathrm{t})=1$. Conversely, at $\mathrm{t}=200 \mathrm{~s}$ (arrows in Fig.5), a modest LF neural modulation was introduced, $\mathrm{N}(\mathrm{t})=1+0.3 \sin (2 \pi 0.1 \mathrm{t})$. This did not entrain the two PVDs, but it was sufficient to disturb phase opposition, disrupt the negative interference and unmask LF oscillations in AP.

As to pulse pressure, note in Fig.5 the presence of a normal systolic/diastolic oscillation in the systemic AP $\left(\mathrm{P}_{1}\right.$, upper panels, upper traces) and its virtual absence in peripheral AP ( $\mathrm{P}_{1}$, upper panels, lower traces) and flows (lower panels): the classical windkessel function which is superimposed to the investigated effect on LF.

\section{Conclusion}

It is proposed that vasomotion, arterial compliances and neural drives are all essential elements in forming LF waves in systemic AP. Probably this perspective would cast new light in the comprehension of the physiological and clinical meaning of short term AP and heart rate variabilities.

\section{References}

[1] Miyakawa K, Polosa C, Koepchen HP, editors. Mechanisms of blood pressure waves. Berlin: Springer Verlag, 1984:3-23.

[2] Malliani A, Pagani M, Lombardi F, Cerutti S. Cardiovascular neural regulation explored in the frequency domain. Circulation 1991; 84:482-92.

[3] Westerhof N, Elzinga G, Sipkema P. An artificial arterial system for pumping hearts. J Appl Physiol 1971; 31:776781.

[4] De Boer RW, Karemaker JM, Strackee J. Hemodynamic fluctuations and baroreflex sensitivity in humans: a beat to beat model. Am J Physiol 1987; 253:680-9.

[5] Seydnejad SR, Kitney RI. Modeling of mayer waves generation mechanisms. IEEE Eng Med Biol Mag 2001; 20:92-100.

[6] Baselli G, Porta A. Simulating the interactions among vasomotion waves of peripheral vascular districts. Computers in Cardiology 2002; 29:49-52.

Address for correspondence.

Giuseppe Baselli

Politecnico di Milano

Dip. di Bioingegneria

P.zza Leonardo da Vinci, 32

20133 Milano, Italy

E-mail address: baselli@biomed.polimi.it 